\title{
On Convolution Dominated Operators
}

\author{
Gero Fendler and Michael Leinert
}

\begin{abstract}
For a locally compact group $G$ we consider the algebra $C D(G)$ of convolution dominated operators on $L^{2}(G)$ : An operator $A: L^{2}(G) \rightarrow$ $L^{2}(G)$ is called convolution dominated if there exists $a \in L^{1}(G)$ such that for all $f \in L^{2}(G)$

$$
|A f(x)| \leq a \star|f|(x) \text { for almost all } x \in G .
$$

In the case of discrete groups those operators can be dealt with quite sufficiently if the group in question is rigidly symmetric. For non-discrete groups we investigate the subalgebra of regular convolution dominated operators $C D_{\text {reg }}(G)$. For amenable $G$ which is rigidly symmetric as a discrete group we show that any element of $C D_{r e g}(G)$ is invertible in $C D_{\text {reg }}(G)$ if it is invertible as a bounded operator on $L^{2}(G)$. We give an example of a symmetric group $E$ for which the convolution dominated operators are not inverse-closed in the bounded operators on $L^{2}(E)$.
\end{abstract}

Mathematics Subject Classification. Primary 47B35; Secondary 43A20.

Keywords. Convolution dominated operators, inverse-closed subalgebras, symmetry.

\section{Introduction}

When one considers a convolution operator on the abelian group $\mathbb{Z}$, then its matrix with respect to the canonical basis of $l^{2}(\mathbb{Z})$ is a Toeplitz matrix, i.e., it is constant along side diagonals. Conversely, a doubly infinite matrix, which is constant along its side diagonals and satisfies certain off-diagonal decay conditions, defines a convolution operator, when it is considered as acting with respect to the above basis. For the class of operators with decay in the sense of $l^{1}$-summability Wiener [44] proved the Fourier transformed version of the following theorem

Theorem 1.1. (Wiener's Lemma) If a two-sided infinite absolutely summable sequence $a=(a(n))_{n \in \mathbb{Z}}$ is invertible as a convolution operator on $l^{2}(\mathbb{Z})$, then the inverse is given by convolution with some $b \in l^{1}(\mathbb{Z})$. 
Based on work of de Leeuw [9] and of Bochner and Phillips [8] on operator-valued Fourier transforms, several authors extended and applied Wiener's lemma to the case of matrices of operators, for which each side diagonal is uniformly bounded and these bounds are summable. The index set always had to be a discrete abelian group or a countable set $[1,16,17,21,42,43]$.

If $G$ is a locally compact abelian group, for simplicity assumed to be compactly generated, then it admits a discrete co-compact subgroup $H$, and $L^{p}(G)=l^{p}\left(H, L^{p}(D)\right)$, where $D$ is some fundamental domain. So vector valued Wiener Lemmata become applicable to classes of integral operators. Baskakov in [2-5] derives results for some of these. Shin and Sun in [41] give an account of those techniques.

If $\mathcal{B}$ is a Banach algebra and $\mathcal{A}$ a subalgebra then $\mathcal{A}$ is called spectral in $\mathcal{B}$ when every element of $\mathcal{A}$ has the same spectrum in $\mathcal{A}$ as it has in $\mathcal{B}$. In $[26,27]$ Kurbatov shows among other results that for a locally compact abelian group the algebra of convolution dominated operators (see 2.1) is spectral in the bounded operators on $L^{p}(G)$. Farrell and Strohmer [12] extend this result to generalised Heisenberg groups (with compact center).

Plenty of work has been done with regularity assumptions on the kernel of an integral operator. Let us just mention interesting studies by Gröchenig and Klotz [18-20,24,25] on norm controlled inverse closedness of smoothness algebras in symmetric algebras. A comprehensive discussion of this important theme would go beyond the scope of this note.

In [13], together with Gröchenig we addressed the discrete nonabelian case using tools from abstract harmonic analysis to circumvent the restrictions of abelian Fourier transformation:

Theorem 1.2. Let $G$ be a discrete finitely generated group of polynomial growth. If a matrix $A$ indexed by $G$ satisfies the off-diagonal decay condition

$$
|A(x, y)| \leq a\left(x y^{-1}\right), \quad x, y \in G
$$

for some $a \in \ell^{1}(G)$ and $A$ is invertible on $\ell^{2}(G)$, then there exists $b \in \ell^{1}(G)$ such that

$$
\left|A^{-1}(x, y)\right| \leq b\left(x y^{-1}\right), \quad x, y \in G,
$$

i. e. the algebra of matrices satisfying (1) is inverse-closed in $B\left(L^{2}(G)\right)$, the bounded operators on $L^{2}(G)$.

Throughout this paper it is assumed that the involution of a Banach *-algebra is isometric. We recall the main line of the proof of this theorem, since this will be a guideline for us.

1. The matrices satisfying (1) form a Banach $*$-algebra. We denote it by $C D(G)$.

2. Identify $C D(G)$ with $l^{1}\left(G, l^{\infty}(G), T\right)$, a twisted $l^{1}$-algebra in the sense of Leptin $[28,29]$.

3. Based essentially on work of Leptin and Poguntke, in particular [35], we proved that $C D(G)$ is symmetric Banach $*$-algebra. 
4. In a final step one relates the symmetry of a Banach $*$-algebra to the invertibility of certain of its elements. This is done with the help of Hulanicki's Lemma [22].

Definition 1.3. $\quad$ 1. A Banach $*$-algebra $A$ is called symmetric if for all $a \in A$

$$
\sigma_{A}\left(a^{*} a\right) \subset[0, \infty),
$$

where $\sigma_{A}\left(a^{*} a\right)$ denotes the spectrum of $a^{*} a$ in $A$.

2. Accordingly, a locally compact group $G$ is called symmetric, if $L^{1}(G)$ is a symmetric Banach *-algebra.

3. A locally compact (l.c.) group $G$ is called rigidly symmetric if for any $C^{*}$ algebra $A$ the Banach *-algebra $L^{1}(G) \hat{\otimes} A$ is symmetric, where the projective tensor product $L^{1}(G) \hat{\otimes} A$ is endowed with its natural $*$-algebra structure.

In these notes we derive variants of Theorem 1.2 for integral operators on non-discrete locally compact groups. This extension follows the ideas of the discrete case in [13], but requires non-trivial modifications. The special problem to be addressed is the measurability and integrability of certain kernels. We extend the results presented in the exposition [14] and provide proofs for them. Meanwhile some of our results have been reproved by Beltiţă and Beltiţă $[6,7]$ and by Măntoiu [36] in the discrete case. After archiving (on arxiv) this paper A. R. Schep kindly informed us that our Proposition 2.3 is a special case of a theorem in his dissertation [40].

It is not known yet if a symmetric group is already rigidly symmetric. An interesting example is the real $a x+b$ group $E$, which is known to be a symmetric group but not symmetric as a discrete group [23]. We shall discuss a specific example of a non-symmetric algebra related to $E$ showing non-symmetry of $C D_{\text {reg }}(E)$. So, in particular, $C D_{\text {reg }}(E)$ is not inverse-closed in $B\left(L^{2}(E)\right)$.

\section{Convolution Dominated Operators}

Let $G$ be a locally compact group.

Definition 2.1. A bounded operator $A: L^{2}(G) \rightarrow L^{2}(G)$ is called a convolution dominated operator if there exists $a \in L^{1}(G)$ such that for all $f \in L^{2}(G)$

$$
|A f(x)| \leq a \star|f|(x) \text { for almost all } x \in G .
$$

We define a norm on the space of convolution dominated operators by

$$
\|A\|_{C D}=\inf \left\{\|a\|_{1}:(2) \text { holds true }\right\}
$$

and denote by $C D$ the normed linear space of convolution dominated operators.

It is clear that in this definition necessarily $a \geq 0$ locally almost everywhere and that the $C D$-norm dominates the operator norm, in fact for 
$1 \leq p \leq \infty$ and $a \in L^{1}(G)$ satisfying $(2)$

$$
\begin{aligned}
\|A f\|_{p} & =\left(\int_{G}|A f|^{p}(x) d x\right)^{1 / p} \leq\left(\int_{G}(a \star|f|)^{p} d x\right)^{1 / p} \\
& \leq\|\lambda(a)\|\|f\|_{p} \leq\|a\|_{1}\|f\|_{p} .
\end{aligned}
$$

It follows that $A \in C D$ extends to a bounded operator on $L^{p}(G), 1 \leq p<\infty$ by continuity, and by duality to a bounded operator on $L^{\infty}(G)$ too.

Proposition 2.2. With the involution of operators on $L^{2}(G)$ and composition of operators as product the space of convolution dominated operators is a Banach *-algebra.

Proof. Let $A, B$ be convolution dominated operators and choose $a$, resp. $b \in$ $L^{1}(G)$ according to $(2)$. Then for $f \in L^{2}(G)$ :

$$
|A \circ B f|(x) \leq a \star|B f|(x) \leq a \star b \star|f|(x) .
$$

From this and because $L^{1}(G)$ is an normed algebra under convolution it is clear that

$$
\|A \circ B\|_{C D} \leq\|A\|_{C D}\|B\|_{C D} .
$$

To see that the involution preserves the space $C D$ take $a \in L^{1}(G)$ according to $(2)$ and $f, h \in L^{2}(G)$ :

$$
\begin{aligned}
\left|\int_{G} A^{*} h(x) \overline{f(x)} d x\right| & =\left|\left(A^{*} h, f\right)\right| \\
& =|(h, A f)| \\
& =\left|\int_{G} h(x) \overline{A f(x)} d x\right| \\
& \leq \int_{G}|h(x)||A f(x)| d x \\
& \leq \int_{G}|h(x)| a \star|f|(x) d x \\
& =\int_{G} a^{*} \star|h|(x)|f|(x) d x,
\end{aligned}
$$

where $a^{*}(x)=\overline{a\left(x^{-1}\right)} \Delta\left(x^{-1}\right)$ is the involution on $L^{1}(G)$, and here $a^{*}(x)=$ $a\left(x^{-1}\right) \Delta\left(x^{-1}\right)$, since $a \geq 0$. Hence, $\left|A^{*} h(x)\right| \leq a^{*} \star|h|(x)$ locally almost everywhere for all $h \in L^{2}(G)$, and it follows that $A^{*} \in C D$ with $\left\|A^{*}\right\|_{C D} \leq$ $\inf \left\{\|a\|_{1}:(2)\right.$ holds true $\}=\|A\|_{C D}$.

To show that $C D$ is a complete space we let $\left(A_{i}\right)_{i \in \mathbb{N}}$ be a sequence in $C D$ with $\sum_{i \in \mathbb{N}}\left\|A_{i}\right\|_{C D}$ convergent. Then we find $a_{i} \in L^{1}(G)$ such that $\left|A_{i} f(x)\right| \leq a_{i} \star|f|(x)$ for almost all $x \in G$ and $\left\|a_{i}\right\|_{1} \leq\left\|A_{i}\right\|_{C D}+2^{-i}$. For $A=\sum_{i \in \mathbb{N}} A_{i}$ this sum is convergent in the space of bounded operators, hence for $f \in L^{2}(G)$ and for a subsequence of the partial sums we have $A f(x)=$ $\lim _{k} \sum_{i=1}^{j_{k}} A_{i} f(x)$ almost everywhere. So $|A f|(x) \leq \sum_{i \in \mathbb{N}}\left|A_{i} f\right|(x) \leq \sum_{i \in \mathbb{N}}$ $a_{i} \star|f|(x)=a \star|f|(x)$. This shows $A \in C D$ with $\|A\|_{C D} \leq\|a\|_{1}$. 
Proposition 2.3. For a given convolution dominated operator $A$ there exists a locally integrable function $F_{A}: G \times G \rightarrow \mathbb{C}$ such that for all $f \in C_{c p}(G)$

$$
A f(x)=\int_{G} F_{A}(x, y) f(y) d y \quad \text { almost everywhere. }
$$

Proof. let $K, K^{\prime} \subset G$ be compact sets and consider on $C(K) \times C\left(K^{\prime}\right)$ the form

$$
\langle A f, h\rangle:=\int_{G} A f(x) h(x) d x,
$$

where $f$ and $h$ are extended as $L^{2}$-functions vanishing outside $K$ respectively $K^{\prime}$. Take $\varphi \in C_{c p}(G), \varphi \geq 0$, then for $f_{1}, \ldots, f_{n} \in C(K)$ and $h_{1}, \ldots, h_{n} \in$ $C\left(K^{\prime}\right)$

$$
\begin{aligned}
& \left|\sum_{i=1}^{n}\left\langle\lambda(\varphi) \circ A f_{i}, h_{i}\right\rangle\right|=\left|\int_{G} \sum_{i=1}^{n} A f_{i}(x)\left(\varphi^{*} \star h_{i}\right)(x) d x\right| \\
& =\left|\int_{G} \int_{G} A\left(\sum_{i=1}^{n} f_{i} h_{i}\left(z^{-1}\right)\right)(x) \varphi^{*}(x z) d z d x\right| \\
& \leq \int_{G} \int_{G}\left(a \star\left|\sum_{i=1}^{n} f_{i} h_{i}\left(z^{-1}\right)\right|\right)(x) \varphi^{*}(x z) d z d x \\
& =\int_{G} \int_{G} \int_{G} a(x y)\left|\sum_{i=1}^{n} f_{i}\left(y^{-1}\right) h_{i}\left(z^{-1}\right)\right| \varphi^{*}(x z) d y d z d x \\
& =\int_{G} \int_{G} \int_{G} a\left(x y^{-1}\right) \Delta\left(y^{-1}\right) \\
& \cdot\left|\sum_{i=1}^{n} f_{i}(y) h_{i}(z)\right| \varphi^{*}\left(x z^{-1}\right) \Delta\left(z^{-1}\right) d y d z d x \\
& \leq \int_{G(y, z) \in K \times K^{\prime}} \sup _{i=1}\left|\sum_{i}^{n} f_{i}(y) h_{i}(z)\right| \\
& \int_{K^{\prime}} \int_{K} a\left(x y^{-1}\right) \Delta\left(y^{-1}\right) \varphi^{*}\left(x z^{-1}\right) \Delta\left(z^{-1}\right) d y d z d x \\
& \leq\|a\|_{1} \sup _{(y, z) \in K \times K^{\prime}}\left|\sum_{i=1}^{n} f_{i}(y) h_{i}(z)\right| \\
& \int_{G} \int_{K^{\prime}} \varphi^{*}\left(x z^{-1}\right) \Delta\left(z^{-1}\right) d z d x \\
& =\|a\|_{1} \sup _{(y, z) \in K \times K^{\prime}}\left|\sum_{i=1}^{n} f_{i}(y) h_{i}(z)\right|\left|K^{\prime}\right| \int_{G} \varphi(x) d x
\end{aligned}
$$

where $\Delta$ denotes the modular function of $G, \varphi^{*}(z)=\varphi\left(z^{-1}\right) \Delta\left(z^{-1}\right)$ and we write $\left|K^{\prime}\right|$ for the left Haar measure of the set $K^{\prime}$. We take an approximate 
unit of $L^{1}(G)$ consisting of functions $\varphi$ as above. Then we see that

$$
\left|\sum_{i=1}^{n}\left\langle A f_{i}, h_{i}\right\rangle\right| \leq\left|K^{\prime}\right|\|a\|_{1} \sup _{(y, z) \in K \times K^{\prime}}\left|\sum_{i=1}^{n} f_{i}(y) h_{i}(z)\right| .
$$

Hence $f \otimes h \mapsto\langle A f, h\rangle$ extends to a linear form on the injective tensor product $C(k) \otimes_{\epsilon} C\left(K^{\prime}\right)=C\left(K \times K^{\prime}\right)$, so there is a Borel measure $\mu$ such that for $f_{1}, \ldots, f_{n} \in C(K)$ and $h_{1}, \ldots, h_{n} \in C\left(K^{\prime}\right)$

$$
\int_{G} \sum_{i=1}^{n} A f_{i}(z) h_{i}(z) d z=\int_{K} \int_{K^{\prime}} \sum_{i=1}^{n} f_{i}(y) h_{i}(z) d \mu(y, z),
$$

and by a computation much similar to the first part of the above one

$$
\left|\int_{K} \int_{K^{\prime}} \sum_{i=1}^{n} f_{i}(y) h_{i}(z) d \mu(y, z)\right| \leq \int_{G} \int_{G} a\left(z y^{-1}\right) \Delta\left(y^{-1}\right)\left|\sum_{i=1}^{n} f_{i}(y) h_{i}(z)\right| d y d z .
$$

This last inequality extends to bounded measurable functions, and if $H \subset$ $K \times K^{\prime}$ is the characteristic function of a set of Haar measure 0 in $G \times G$, then by Fubini's theorem, for almost all $z \in G$ the function $H(., z)$ vanishes off a set of measure zero. The above estimate implies

$$
0 \leq \int H d \mu \leq \int_{G} \int_{G} a\left(z y^{-1}\right) \Delta\left(y^{-1}\right) H(y, z) d y d z=0 .
$$

That is, $\mu$ is absolutely continuous with respect to the Haar measure on $G \times G$, and by the Radon Nikodym theorem there exists a kernel $F_{A}^{\left(K^{\prime}, K\right)} \in$ $L^{1}\left(K \times K^{\prime}\right)$ such that

$$
\int_{G} A f(x) h(x) d x=\int_{K^{\prime}} \int_{K} F_{A}^{\left(K^{\prime}, K\right)}(x, y) f(y) h(x) d y d x
$$

whenever $f, h$ are continuous with support in $K$ resp. $K^{\prime}$. It is now a standard procedure to check the consistency of these kernels for different pairs of compact sets so that they define a locally integrable kernel $F_{A}$ on $G \times G$, which represents $A$ as claimed in the proposition.

Remark 2.4. In the above proof we have seen that in terms of the kernel $F_{A}$ of a convolution dominated operator $A$ the inequality (2) may be rewritten as

$$
F_{A}(x, y) \leq a\left(x y^{-1}\right) \Delta\left(y^{-1}\right) \quad \text { locally almost everywhere (l.a.e.) }
$$

Remark 2.5. Conversely, if $F: G \times G \rightarrow \mathbb{C}$ is a locally integrable function, such that for some $a \in L^{1}(G)$ :

$$
|F(x, y)| \leq a\left(x y^{-1}\right) \Delta\left(y^{-1}\right),
$$

then by

$$
A f(x)=\int_{G} F(x, y) f(y) d y
$$

a bounded operator on $L^{2}(G)$ can be defined, which clearly is dominated by $\lambda(a)$. For this it suffices to check the Schur conditions

$$
\operatorname{ess}_{\sup _{x}} \int|F(x, y)| d y<\infty \quad \text { and } \quad \operatorname{ess} \sup _{y} \int|F(x, y)| d x<\infty
$$


Remark 2.6. The kernel of a convolution dominated operator satisfies the Schur conditions and hence represents the operator in the sense that for all $f \in L^{2}(G)$ the following integral converges l.a.e. and

$$
A f(x)=\int_{G} F_{A}(x, y) f(y) d y \quad \text { l.a.e. }
$$

Now let $a_{i}, i \in \mathbb{N}$ be a sequence such that (3) holds true for each $i$ and such that $\left\|a_{i}\right\|_{1} \leq\|A\|_{c d}+2^{-i}$. Then, for $n \in \mathbb{N}$, let $b_{n}=a_{1} \wedge \cdots \wedge a_{n}$, where $\wedge$ denotes the operation of taking the pointwise minimum of integrable functions. The functions $b_{n}$ are bounded below, and form a decreasing sequence in $L^{1}(G)$. Thus they converge to $b$, say. It is easily seen from (3) that this limit satisfies (2), furthermore $\|b\|_{1}=\|A\|_{C D}$. We conclude:

Remark 2.7. The infimum in Definition 2.1 is attained.

If $G$ is a discrete group then an element $A \in C D$ may be represented uniquely by its matrix with respect to the basis given by the unit masses placed at the group elements

$$
A(x, y)=\left(A \delta_{y} \mid \delta_{x}\right)
$$

Denote $m_{z}$ as the $z$-th side-diagonal of the matrix. Then the matrix is the direct sum of its side diagonals and therefore

$$
A=\sum_{z \in G} \lambda(z) \circ D^{m_{z}},
$$

where $D^{m} \in B\left(l^{2}(G)\right)$ is the multiplication operator with $m \in l^{\infty}(G)$. In [13] this was used to show that

$$
R: \ell^{1}\left(G, \ell^{\infty}(G), T\right) \rightarrow B\left(\ell^{2}(G)\right)
$$

defined by

$$
R: \sum_{z \in G} \delta_{z} \otimes m_{z} \mapsto \sum_{z \in G} \lambda(z) \circ D^{m_{z}} .
$$

is surjective from a certain twisted $L^{1}$-algebra onto $C D$. In fact this map is an isometric $*$-algebra isomorphism. We shall next define the twisted $L^{1}$-algebra and the map $R$, which unfortunately is no longer surjective.

Let $\operatorname{Ruc}(G)$ denote the space of bounded right uniformly continuous functions, i. e. those $F \in L^{\infty}(G)$ such that ess $\sup _{x \in G}\left|f\left(y^{-1} x\right)-f(x)\right| \rightarrow$ 0 as $y \rightarrow 0$. (This definition of right uniform continuity follows [39, Ch. 3, 1.8(vi)]) This space is just the subspace of $L^{\infty}$ of those elements on which left translation acts norm continuously. It is a closed subspace containing only continuous functions.

For $y \in G$ denote $T_{y}$ left translation on $\operatorname{Ruc}(G)$, that is $T_{y} n(z)=$ $n\left(y^{-1} z\right), n \in R u c(G)$. We consider the map $T: y \mapsto T_{y}$ as a homomorphism of $G$ into the group of isometric automorphisms of the $C^{*}$-algebra $\operatorname{Ruc}(G)$, which is continuous when the latter group is endowed with the strong operator topology. With this homomorphism we form the twisted $L^{1}$-algebra $\mathcal{L}=$ $L^{1}(G, R u c(G), T)$ in the sense of Leptin [28-30]. The underlying Banach space 
of $\mathcal{L}$ is the space of $\operatorname{Ruc}(G)$-valued Bochner integrable functions on $G$, but we will often interpret it as the projective tensor product

$$
L^{1}(G, \operatorname{Ruc}(G))=L^{1}(G) \hat{\otimes} \operatorname{Ruc}(G) .
$$

Thus for an element $f \in L^{1}(G, R u c(G))$ we denote its value in $R u c(G)$ by $f(x), x \in G$, and we write $f(x)(z)$ or $f(x, z)$ for the value of this $R u c$-function at $z \in G$.

The twisted convolution of $h, f \in \mathcal{L}$ is defined by

$$
h \star f(x)=\int_{G} T_{y} h(x y) f\left(y^{-1}\right) d y, \quad \text { for } x \in G,
$$

and the involution of $h \in \mathcal{L}$ by

$$
h^{*}(x)=\Delta\left(x^{-1}\right) \overline{T_{x^{-1}}\left(h\left(x^{-1}\right)\right)}, \quad \text { for } x \in G .
$$

The properties of the projective tensor product ensure that $R: a \otimes m \mapsto$ $\lambda(a) \circ D^{m}$ extends to a norm-nonincreasing linear map from $L^{1}(G, R u c(G))$ to $B\left(L^{2}(G)\right)$.

Proposition 2.8. The map $R: L^{1}(G, R u c(G), T) \rightarrow B\left(L^{2}(G)\right)$ is an *-algebra homomorphism with range in $C D$. It is isometric from $L^{1}(G, R u c(G), T)$ into $C D$.

Proof. Since $\lambda$ is a continuous unitary representation of $G$ on $L^{2}(G)$ and $D: m \mapsto D^{m}$ is a *-representation of $R u c$ in $B\left(L^{2}(G)\right)$, with $\lambda(x)^{*} \circ D^{m} \circ$ $\lambda(x)=D_{x}^{T^{-1} m}, \forall x \in G, m \in R u c$, [30, Satz 3] shows that $R$ defines a nondegenerate $*$-representation of $\mathcal{L}$.

For $a \otimes m \in L^{1}(G) \hat{\otimes} \operatorname{Ruc}(G)$ and $h \in L^{2}(G)$ we have

$$
|R f(x)|=|\lambda(a)(m h)(x)| \leq\|m\|_{\infty}|a| \star|f|(x) .
$$

This shows that $R$ maps into CD and does not increase the norm as a map from $\mathcal{L}$ to $C D$.

Now assume that for $f \in L^{1}(G, \operatorname{Ruc}(G), T)$ we have $a \in L^{1}(G)$ such that for all $h \in L^{2}(G):|R f(h)|(x) \leq a \star|h|(x)$. Then

$$
\begin{aligned}
\int_{G} a\left(x y^{-1}\right) \Delta\left(y^{-1}\right)|h(y)| d y & =a \star|h|(x) \\
& \geq|R f(h)|(x) \\
& =\left|\int_{G} \lambda(y)(f(y)(.) h(.))(x) d y\right| \\
& =\left|\int_{G} f(y)\left(y^{-1} x\right) h\left(y^{-1} x\right) d y\right| \\
& =\left|\int_{G} f\left(x y^{-1}\right)(y) \Delta\left(y^{-1}\right) h(y) d y\right| .
\end{aligned}
$$

Then $\left|f\left(x y^{-1}\right)(y)\right| \leq a\left(x y^{-1}\right) \Delta\left(y^{-1}\right)$ for almost all $(x, y) \in G \times G$, or $|f(x)(y)| \leq a(x)$ for almost all $(x, y) \in G \times G$. It follows that $\|f(x)\|_{\infty} \leq a(x)$ for almost all $x \in G$. Hence

$$
\|f\|_{L^{1}(G, R u c(G), T)}=\int_{G}\|f(x)\|_{\infty} d x \leq\|a\|_{1} .
$$


As a consequence: $R: L^{1}(G, \operatorname{Ruc}(G), T) \rightarrow C D$ is isometric.

Definition 2.9. Elements in the image of $R$ we call regular convolution dominated operators, and denote the whole image by $C D_{\text {reg }}$.

The continuous functions vanishing at infinity $C_{0}(G)$ are a closed twosided ideal in $\operatorname{Ruc}(G)$ and as is easily seen this implies that $L^{1}\left(G, C_{0}(G), T\right)$ is a closed two-sided ideal in $L^{1}(G, \operatorname{Ruc}(G), T)$.

Remark 2.10. It follows from [35, Theorem 4] that $L^{1}\left(G, C_{0}(G), T\right)$ is simple and symmetric. The representation $\rho$ in the beginning of their proof, we denoted it by $R$, maps $L^{1}\left(G, C_{0}(G), T\right.$ ) into an ideal (in $C D_{\text {reg }}$ ) of compact operators. Moreover the operator norm closure of $R\left(L^{1}\left(G, C_{0}(G), T\right)\right)$ equals the compact operators [34].

\section{Symmetry of the Twisted $L^{1}$-Algebra}

In this section we shall show that the twisted $L^{1}$-algebra $L^{1}(G, R u c(G), T)$ is a symmetric Banach *-algebra. To this end we shall first recall a criterion for the symmetry of a Banach *-algebra.

Definition 3.1. Let $E$ be a normed linear space and $\mathcal{A}$ be a Banach $*$-algebra. A representation $\rho: \mathcal{A} \rightarrow \operatorname{End}(E)$ is called preunitary if there exists a Hilbert space $H$ and a bounded $*$-representation $\pi: \mathcal{A} \rightarrow B(H)$ together with an injective and bounded operator $U: E \rightarrow H$ intertwining $\rho$ and $\pi$.

$$
U \circ \rho(a)=\pi(a) \circ U, \quad \forall a \in \mathcal{A} .
$$

Remark 3.2. If $\rho$ is a contractive representation of $A$ on a Banach space $E$ then we are given a Banach $A$ module in the sense of Leptin. The representation is preunitary in the above sense if the Banach $A$ module is preunitary in the sense of Leptin [33].

It is clear that the image, under $U$, of a $\rho(\mathcal{A})$-invariant subspace is invariant under $\pi(\mathcal{A})$. We may and do assume that $U(E)$ is dense in $\mathrm{H}$.

The following question appears naturally. If $\rho$ is an algebraically irreducible, preunitary representation, can the representation $\pi$ in the definition be chosen topologically irreducible? The answer is positive:

Proposition 3.3. Let $A$ be a Banach *-algebra with approximate identity. If $\rho: A \rightarrow B(E)$ is an algebraically irreducible, preunitary representation of $A$ then there is a topologically irreducible $*$ representation $\pi$ extending $\rho$.

Proof. The representation $\rho$ is preunitary, hence its kernel is a *-ideal, and possibly replacing $A$ by $A / \operatorname{kern}(\rho)$ we may assume that $\rho$ is faithful. Since $\rho: A \rightarrow B(E)$ is algebraically irreducible there is a maximal modular left ideal $M \subset A$, with modular right unit $u$, such that $E$ and $A / M$ are algebraically isomorphic, and $\rho$ appears as left multiplication on $A / M$. For $b \in A$ denote $\bar{b}$ its class in $A / M$ and let

$$
(\mid): A / M \times A / M \rightarrow \mathbb{C}
$$


be the positive sesquilinear form given by

$$
(\bar{b} \mid \bar{c})=(U(\bar{b}) \mid U(\bar{c}))_{H}
$$

The functional

$$
\phi(a)=(a \bar{u} \mid \bar{u})=(\pi(a) U(\bar{u}) \mid U(\bar{u}))_{H}, \quad a \in A
$$

is non-trivial, positive and continuous with respect to the maximal $C^{*}$-norm of $A$.

Let $C$ denote the $C^{*}$-hull of $A$ and $\bar{M}$ the closure of $M$ in $C$. For $a \in M$ we have $\phi(a)=0$ hence $\bar{M} \subset \operatorname{kern}(\phi)$, so $\bar{M} \neq C$. Let $N \supset M$ be a proper maximal modular left ideal in $C$ containing $M$.

First we claim that $N \cap A=M$. By definition of $N$ we only have to show $N \cap A \subset M$. Clearly $N \cap A$ is a left ideal in $A$, and $u$ a right modular unit. Note that $N \cap A$ is a proper ideal in $A$ since $N$ is a proper ideal in $C$. By the maximality of $M$ it follows that $N \cap A \subset M$.

Now by $[11,2.9 .5]$ there is a pure state $\psi$ on $C$ such that $N=\{b \in$ $\left.C: \psi\left(b^{*} b\right)=0\right\}$. Hence $M=N \cap A=\left\{a \in A: \psi\left(a^{*} a\right)=0\right\}$. So the GNS representation of $A$ constructed from the (pure state) $\psi_{\mid A}$ is a topologically irreducible extension of $\rho$ containing $E$ as a dense invariant subspace.

Since an algebraically irreducible representation of a Banach *-algebra is equivalent to a contractive one, we may use from [32]:

Theorem 3.4. A Banach *-algebra is symmetric if and only if all its nontrivial algebraically irreducible representations are preunitary.

Following the concept used in the discrete case [13] one would like to define a map

$$
Q: L^{1}(G, R u c(G), T) \rightarrow L^{1}(G) \hat{\otimes} B\left(L^{2}(G)\right)
$$

by

$$
Q(f)=\left\{x \mapsto \lambda(x) \circ D^{f(x)}\right\} .
$$

But this does not work since $Q(f)$ is not Bochner measurable because $\lambda$ : $G \rightarrow B\left(L^{2}(G)\right)$, is strongly continuous, but not norm continuous.

The problem can be worked around by showing, completely analogously to [37], that algebraically irreducible representations of certain twisted $L^{1}$ algebras of $G$ remain irreducible when "restricted" to the discretised group.

So, as before let $G$ be a locally compact group, $\mathcal{A}$ a Banach *-algebra, with isometric involution and a left approximate identity. Further we assume that $T: G \rightarrow \operatorname{Aut}(\mathcal{A})$ is a continuous homomorphism from $G$ into the group of $*$-automorphisms of $\mathcal{A}$, where $\operatorname{Aut}(\mathcal{A})$ is endowed with the strong operator topology, i.e. $y \mapsto T_{y} a$ is continuous from $G$ to $\mathcal{A}$, for all $a \in \mathcal{A}$. With these data we form the twisted $L^{1}$-algebra (as above) $\mathcal{L}=L^{1}(G, \mathcal{A}, T)$.

Let $E$ be a nontrivial linear space and let $\rho: \mathcal{L} \rightarrow B(E)$ be a non-trivial algebraically irreducible representation on it. Given $\xi_{0} \in E, \xi_{0} \neq 0$, one has a norm on $E$ :

$$
\|\xi\|_{E}=\inf \left\{\|f\|_{\mathcal{L}}: f \in \mathcal{L}, \rho(f) \xi_{0}=\xi\right\}
$$


with respect to which $E$ is a complete space. In fact it is the quotient of $\mathcal{L}$ with respect to the maximal modular left ideal $\left\{f \in \mathcal{L}: \rho(f) \xi_{0}=0\right\}$. Different $\xi_{0} \in E$ define different but equivalent norms.

As in the proof of [30, Satz 3], $\rho: G \rightarrow B(E)$ and $\rho: \mathcal{A} \rightarrow B(E)$ are representations of the group respectively of the Banach algebra $\mathcal{A}$. The operators $\rho(x), x \in G$, are isometries and $\rho$ does not increase norms. Here the operators $\rho(x), x \in G$ and $\rho(a), a \in \mathcal{A}$ do not necessarily commute, but we have the relation

$$
\rho\left(y^{-1}\right) \rho(a) \rho(y)=\rho\left(T_{y^{-1}} a\right),
$$

and furthermore for all $f \in L^{1}(G, \mathcal{A}, T), \xi \in E$

$$
\rho(f) \xi=\int_{G} \rho(x) \rho(f(x)) \xi d x .
$$

Unfortunately the proof in [30] is done with the hypothesis of dealing with a $*$-representation. Apart from some algebraic identities the main ingredient is [30, Satz 2] in its consequence (1.2) loc.cit..

Now we take the group $G$ with the discrete topology, denote it $G_{d}$. We form the twisted $L^{1}$-algebra $\mathcal{L}_{d}:=l^{1}(G, \mathcal{A}, T)$, and define a representation of it on $E$ by

$$
\rho_{d}(h) \xi=\sum_{x \in G} \rho(x) \rho(h) \xi, \quad \xi \in E, \quad h \in \mathcal{L}_{d}
$$

Lemma 3.5. Let $\rho$ be an algebraically irreducible representation of $L^{1}(G, \mathcal{A}, T)$ and assume the above settings. Then the representation $\rho_{d}: l^{1}(G, \mathcal{A}, T) \rightarrow$ $B(E)$ is algebraically irreducible.

Proof. We follow the proof of [37, Theorem 2]. Assume that $E^{\prime} \subset E$ is a non-trivial $\rho_{d}\left(\mathcal{L}_{d}\right)$ invariant subspace; we have to show that $E^{\prime}=E$. To this end we take a fixed nonzero $\xi_{0} \in E^{\prime}$, and the corresponding norm, see (6), on $E$.

Claim 1. We claim that for $\xi \in E$ and $\varepsilon>0$ there is $h \in l^{1}(G, \mathcal{A}, T)$ such that

$$
\left\|\rho_{d}(h) \xi_{0}-\xi\right\|_{E} \leq \varepsilon \quad \text { and } \quad\|h\|_{\mathcal{L}_{d}} \leq\|\xi\|_{E}+\varepsilon .
$$

The claim implies the assertion of the lemma. For, if $\eta \in E$ is given we have to find $h \in \mathcal{L}_{d}$ such that $\rho_{d}(h) \xi_{0}=\eta$, and this is done inductively as follows: First we find $h_{1} \in \mathcal{L}_{d}$ such that

$$
\left\|\rho_{d}\left(h_{1}\right) \xi_{0}-\eta\right\|_{E} \leq 2^{-1} \quad \text { and } \quad\left\|h_{1}\right\|_{\mathcal{L}_{d}} \leq\|\eta\|_{E}+2^{-1} .
$$

If $h_{1}, \ldots, h_{n}$ are already defined with

$$
\left\|\sum_{i=1}^{n} \rho_{d}\left(h_{i}\right) \xi_{0}-\eta\right\|_{E} \leq 2^{-n}
$$

and

$$
\left\|h_{i}\right\|_{\mathcal{L}_{d}} \leq\left\|\sum_{j=1}^{i-1} \rho_{d}\left(h_{j}\right) \xi_{0}-\eta\right\|_{E}+2^{-i}, \quad i=1, \ldots, n
$$


then we choose $h_{n+1} \in \mathcal{L}_{d}$ such that

$$
\left\|\rho_{d}\left(h_{n+1}\right) \xi_{0}-\left(\eta-\sum_{i=1}^{n} \rho_{d}\left(h_{i}\right) \xi_{0}\right)\right\|_{E} \leq 2^{-(n+1)}
$$

and

$$
\left\|h_{n+1}\right\|_{\mathcal{L}_{d}} \leq\left\|\left(\sum_{i=1}^{n} \rho_{d}\left(h_{i}\right) \xi_{0}-\eta\right)\right\|_{E}+2^{-(n+1)}
$$

Since

$$
\left\|h_{i}\right\|_{\mathcal{L}_{d}} \leq\left\|\sum_{j=1}^{i-1} \rho_{d}\left(h_{j}\right) \xi_{0}-\eta\right\|_{E}+2^{-i} \leq 2^{-(i-1)}+2^{-i} \quad \text { for } i \geq 2,
$$

the sum $\sum_{1}^{\infty} h_{i}$, call it $h$, exists in $\mathcal{L}_{d}$, and

$$
\left\|\rho_{d}(h) \xi_{0}-\eta\right\|_{E}=\lim _{n \rightarrow \infty}\left\|\sum_{i=1}^{n} \rho_{d}\left(h_{i}\right) \xi_{0}-\eta\right\|_{E}=0
$$

It remains to establish the claim. So let $\delta>0$ be a positive real number to be determined later. By definition of the norm on $E$ we find $f \in L^{1}(G, \mathcal{A}, T)$, with

$$
\rho(f) \xi_{0}=\xi \quad \text { and } \quad\|f\|_{\mathcal{L}}<\|\xi\|_{E}+\delta .
$$

Since the space of continuous, compactly supported, $\mathcal{A}$ valued functions $C_{c p}(G, \mathcal{A})$ is dense in $L^{1}(G, \mathcal{A}, T)$ we find, in turn, a function $f_{1} \in C_{c p}(G, \mathcal{A})$ such that

$$
\left\|\rho\left(f_{1}\right) \xi_{0}-\xi\right\|_{E}<\delta \quad \text { and } \quad\left\|f_{1}\right\|_{\mathcal{L}}<\|\xi\|_{E}+\delta
$$

Denote $S$ the support of $f_{1}$ and $|S|$ its Haar measure. Since $\rho$ is a strongly continuous representation of $G$ and since $f$ is uniformly continuous, there is a neighbourhood $U$ of the identity such that

$$
\begin{array}{r}
\left\|\rho(u) \xi_{0}-\xi_{0}\right\|_{E}<\delta, \quad \forall u \in U \quad \text { and } \\
\left\|f_{1}(x u)-f_{1}(x)\right\|_{\mathcal{A}}<\delta|S|^{-1}, \quad \forall x \in G, \quad u \in U .
\end{array}
$$

As $S$ is compact it can be covered by finitely many translates $x_{1} U, \ldots, x_{m} U$ of $U$. We make this covering disjoint $V_{1}:=x_{1} U \cap S$ and inductively $V_{k}:=$ $\left(x_{k} U \cap S\right) \backslash \cup_{j<k} V_{j}$. The $V_{j}$ are measurable pairwise disjoint subsets of $S$, hence $\sum_{1}^{m}\left|V_{j}\right| \leq|S|$.

Now let $a_{j}=f_{1}\left(x_{j}\right) \in \mathcal{A}, j=1, \ldots, m$, and $f_{2} \in L^{1}(G, \mathcal{A}, T)$ be given by

$$
f_{2}=\sum_{j=1}^{m} a_{j} \chi_{V_{j}}
$$


Then

$$
\begin{aligned}
\left\|f_{2}\right\|_{L^{1}(G, \mathcal{A}, T)} & \leq\left\|f_{2}-f_{1}\right\|_{L^{1}(G, \mathcal{A}, T)}+\left\|f_{1}\right\|_{L^{1}(G, \mathcal{A}, T)} \\
& \leq \sum_{j=1}^{m} \int_{V_{j}}\left\|f_{1}(x)-f_{1}\left(x_{j}\right)\right\|_{\mathcal{A}} d x+\|\xi\|_{E}+\delta \\
& \leq \sum_{j=1}^{m}\left|V_{j}\right| \delta|S|^{-1}+\|\xi\|_{E}+\delta \\
& \leq\|\xi\|_{E}+2 \delta .
\end{aligned}
$$

The desired $h \in l^{1}(G, \mathcal{A}, T)$ is defined by

$$
h=\sum_{j=1}^{m} a_{j}\left|V_{j}\right| \delta_{x_{j}} .
$$

Then

$$
\begin{aligned}
\|h\|_{l^{1}(G, \mathcal{A}, T)} & =\sum_{j=1}^{m}\left\|a_{j}\right\|_{\mathcal{A}}\left|V_{j}\right|=\left\|\sum_{j=1}^{m} a_{j} \chi_{V_{j}}\right\|_{L^{1}(G, \mathcal{A}, T)} \\
& \leq\|\xi\|_{E}+2 \delta .
\end{aligned}
$$

Moreover,

$$
\left\|\rho_{d}(h) \xi_{0}-\xi\right\|_{E} \leq\left\|\rho_{d}(h) \xi_{0}-\rho\left(f_{2}\right) \xi_{0}\right\|_{E}+\left\|\rho\left(f_{2}\right) \xi_{0}-\xi\right\|_{E}
$$

The second term can be estimated by

$$
\begin{aligned}
\left\|\rho\left(f_{2}\right) \xi_{0}-\rho\left(f_{1}\right) \xi_{0}\right\|_{E}+\left\|\rho\left(f_{1}\right) \xi_{0}-\xi\right\|_{E} & \leq\left\|f_{1}-f_{2}\right\|_{L^{1}(G, \mathcal{A}, T)}\left\|\xi_{0}\right\|_{E}+\delta \\
& \leq \delta\left\|\xi_{0}\right\|_{E}+\delta .
\end{aligned}
$$

For the first term we use that $\rho: \mathcal{A} \rightarrow B(E)$ is bounded by one and that $s \in V_{j}$ can be written $s=x_{j} u$ with $u \in U$ :

$$
\begin{aligned}
\left\|\rho_{d}(h) \xi_{0}-\rho\left(f_{2}\right) \xi_{0}\right\|_{E} & \leq \sum_{j=1}^{m}\left\|\rho\left(a_{j}\right)\left|V_{j}\right| \rho\left(x_{j}\right) \xi_{0}-\int_{V_{j}} \rho\left(a_{j}\right) \rho(s) \xi_{0} d s\right\|_{E} \\
& \leq \sum_{j=1}^{m}\left\|a_{j}\right\|_{\mathcal{A}}\left\|\int_{V_{j}} \rho\left(x_{j}\right) \xi_{0}-\rho(s) \xi_{0} d s\right\|_{E} \\
& \leq \sum_{j=1}^{m}\left\|a_{j}\right\|_{\mathcal{A}} \int_{V_{j}}\left\|\rho\left(x_{j}\right) \xi_{0}-\rho(s) \xi_{0}\right\|_{E} d s \\
& \leq \sum_{j=1}^{m}\left\|a_{j}\right\|_{\mathcal{A}} \int_{V_{j}} \delta d s \\
& =\delta\left\|f_{2}\right\|_{\mathcal{L}} \leq \delta\left(\|\xi\|_{E}+2 \delta\right)
\end{aligned}
$$

Altogether we found $h \in l^{1}(G, \mathcal{A}, T)$ such that

$$
\|h\|_{l^{1}(G, \mathcal{A}, T)} \leq\|\xi\|_{E}+2 \delta \quad \text { and } \quad\left\|\rho_{d}(h) \xi_{0}-\xi\right\|_{E} \leq \delta\left(\|\xi\|_{E}+2 \delta\right) ;
$$

taking $\delta$ small enough now proves the claim. 
From here onward we assume that $\mathcal{A}$ is a $C^{*}$-algebra and that the operators $T_{y}$ are isometries. We recall that we assumed that they preserve the involution: $T_{y}\left(a^{*}\right)=\left(T_{y} a\right)^{*}, a \in \mathcal{A}, y \in G$. Before discussing the symmetry of $L^{1}(G, \mathcal{A}, T)$ we shall first look at the discretised version.

So let $D: \mathcal{A} \rightarrow B(\mathcal{H})$ be a faithful $*$-representation of $\mathcal{A}$ on some Hilbert space $\mathcal{H}$. We define a map

$$
Q: \ell^{1}\left(G_{d}, \mathcal{A}, T\right) \rightarrow \ell^{1}\left(G_{d}\right) \hat{\otimes} B(\mathcal{H})
$$

by

$$
f=\sum_{v} \delta_{v} \otimes m_{v} \mapsto \sum_{v} \delta_{v} \otimes T_{v} \circ D\left(m_{v}\right)
$$

Proposition 3.6. The map $Q$ is an isometric *-isomorphism of $\ell^{1}\left(G_{d}, \mathcal{A}, T\right)$ onto a closed $*$-subalgebra of $l^{1}\left(G_{d}\right) \hat{\otimes} B(\mathcal{H})$.

Proof. The proof rests on the isometric identification $l^{1}(G, E)=l^{1}(G) \hat{\otimes} E$, which holds for any Banach space $E$ [10, Ch. VIII.1.10]. It follows that for $f=\sum_{v} \delta_{v} \otimes m_{v} \in l^{1}(G, \mathcal{A}, T)$

$$
\begin{aligned}
\|f\|_{1} & =\sum_{v}\left\|m_{v}\right\|_{\mathcal{A}}=\sum_{v}\left\|T_{v} \circ D\left(m_{v}\right)\right\|_{B(\mathcal{H})} \\
& =\left\|\sum_{v} \delta_{v} \otimes T_{v} \circ D\left(m_{v}\right)\right\|_{\ell^{1}(G) \hat{\otimes} B(\mathcal{H})} \cdot
\end{aligned}
$$

Thus $Q$ is an isometry. Let $h=\sum_{v} \delta_{v} \otimes n_{v}$, then

$$
h \star f=\sum_{v} \delta_{v} \otimes l_{v}
$$

where $l_{v}=\sum_{y \in G}\left(T_{y} n_{v y}\right) m_{y^{-1}}$. Hence

$$
\begin{aligned}
Q(h \star f) & =\sum_{v} \delta_{v} \otimes T_{v} D\left(l_{v}\right) \\
& =\sum_{v} \delta_{v} \otimes \sum_{\{z, w: z w=v\}} T_{z} D\left(n_{z}\right) T_{w} D\left(m_{w}\right) \\
& =\sum_{z, w} \delta_{z} \delta_{w} \otimes T_{z} D\left(n_{z}\right) T_{w} D\left(m_{w}\right) \\
& =\left(\sum_{z \in G} \delta_{z} \otimes T_{z} D\left(n_{z}\right)\right)\left(\sum_{w} \delta_{w} \otimes T_{w} D\left(m_{w}\right)\right)=Q(h) Q(f) .
\end{aligned}
$$

Similarly one computes that $Q$ intertwines the involutions. In fact

$$
\begin{aligned}
Q(f)^{*} & =\sum_{v} \delta_{v}^{*} \otimes\left(T_{v} \circ D\left(m_{v}\right)\right)^{*} \\
& =\sum_{v} \delta_{v^{-1}} \otimes T_{v^{-1}} D\left(T_{v} m_{v}{ }^{*}\right) \\
& =\sum_{v^{-1}} \delta_{v} \otimes T_{v} D\left(T_{v^{-1}} m_{v^{-1}}{ }^{*}\right)=Q\left(f^{*}\right) .
\end{aligned}
$$


Thus $Q$ is a $*$-homomorphism. Since $Q$ is an isometry, the image of $Q$ is a closed subalgebra of $l^{1}\left(G_{d}\right) \hat{\otimes} B(\mathcal{H})$.

Because symmetry is inherited by closed subalgebras, we obtain the following consequence.

Corollary 3.7. Let $G$ be a discrete rigidly symmetric group, $\mathcal{A}$ a $C^{*}$-algebra. Then $l^{1}\left(G_{d}, \mathcal{A}, T\right)$ is a symmetric Banach *-algebra. In particular any of its algebraically irreducible representations is preunitary.

Theorem 3.8. If $G$ is rigidly symmetric as a discrete group, and if $\mathcal{A}$ is a $C^{*}$-algebra, then $L^{1}(G, \mathcal{A}, T)$ is symmetric.

Proof. We shall verify that a non-trivial algebraically irreducible representation $\rho: L^{1}(G, \mathcal{A}, T) \rightarrow \operatorname{End}(E)$ is preunitary. We know that its discretised version $\rho_{d}: l^{1}\left(G_{d}, \mathcal{A}, T\right) \rightarrow E n d(E)$ is preunitary too. So let $H$ be the Hilbert space, $\pi_{d}$ the $*$ - representation of $l^{1}\left(G_{d}, \mathcal{A}, T\right)$ on it, and $U$ the intertwining operator according to the Definition 3.1. Now $E$ is a complete space with respect to the norm

$$
\|\xi\|_{d}=\inf \left\{\|h\|_{l^{1}\left(G_{d}, \mathcal{A}, T\right)}: h \in l^{1}\left(G_{d}, \mathcal{A}, T\right), \rho(h) \xi_{0}=\xi\right\},
$$

as well as with respect to \|\|$_{E}$. A slight modification of the argument given after Claim 1 shows that \|\|$_{E} \leq 2\|\|_{d}$, hence these norms are equivalent. Furthermore, $h \mapsto \pi_{d}(h) U \xi_{0}=U \rho_{d}(h) \xi_{0}$ is bounded from $l^{1}\left(G_{d}, \mathcal{A}, T\right)$ to $H$. These two facts show that $U: E \rightarrow H$ is bounded.

From [30, Satz 3] we know that there is a unitary representation $\pi$ : $G_{d} \rightarrow B(H)$ and a $*$-representation (again denoted by the same letter) $\pi$ : $\mathcal{A} \rightarrow B(H)$ such that

$$
\pi\left(x^{-1}\right) \pi(a) \pi(x)=\pi\left(T_{x^{-1}} a\right), \quad \forall x \in G, \quad a \in \mathcal{A}
$$

and

$$
\pi_{d}(h)=\sum_{x \in G} \pi(x) \pi(h(x)), \quad \forall h \in l^{1}\left(G_{d}, \mathcal{A}, T\right) .
$$

Since $\rho: G \rightarrow B(E)$ is a continuous representation and $U$ a continuous intertwining operator it follows that by means of $\pi$ the group $G$ acts continuously on the image $U(E)$ in $H$. As this subspace is dense in $H$ and $\pi$ is a bounded representation we infer that this action is continuous on the whole space $H$. This allows to define a representation $\pi$ of $\mathcal{L}=L^{1}(G, \mathcal{A}, T)$ on $H$ by

$$
\pi(f) \eta=\int_{G} \pi(x) \pi(f(x)) \eta d x \quad \eta \in H, \quad f \in \mathcal{L} .
$$

It is easily checked that this formula defines a $*$-representation of $\mathcal{L}$ on $H$, and that $U$ intertwines $\pi$ and $\rho$.

Corollary 3.9. If $G$ is rigidly symmetric as a discrete group then the twisted algebra $L^{1}(G, R u c(G), T)$ is a symmetric Banach *-algebra.

Remark 3.10. (i) Locally compact nilpotent groups are rigidly symmetric, even as discrete groups [37, Corollary 6]. 
(ii) If for the settings of $[35$, Theorem 5] we choose $G=H=\mathbb{R}$ with the action $\omega:(s, x) \mapsto x^{s}:=e^{s} x$ of the additive group $\mathbb{R}$ on itself and $\mathcal{D}=C_{0}(\mathbb{R})$, then we see that $L^{1}(\mathbb{R}, \mathcal{D})$, with the trivial action of $\mathbb{R}$ on $\mathcal{D}$ is symmetric, whereas $L^{1}\left(\mathbb{R}, L^{1}(\mathbb{R}, \mathcal{D}), \tilde{T}\right)$, with action

$$
\tilde{T}(s) f(x)(\cdot)=e^{-s} f(\omega(-s, x))(\omega(s, \cdot)) \in \mathcal{D}, \quad f \in L^{1}(\mathbb{R}, \mathcal{D}), \quad s, x \in \mathbb{R}
$$

is not symmetric. Hence in the above theorem it is necessary to assume that $\mathcal{A}$ is a $C^{*}$-algebra and not only a symmetric Banach-*-algebra. See the example in Sect. 5 .

(iii) A special role in the theory of symmetry of group algebras is played by the group of affine mappings of the real line, the " $a x+b$ " -group, denoted $E$. This group has a symmetric $L^{1}$-algebra [31], but its discretised version is not symmetric [23]. We do not know about the rigid symmetry of the continuous group.

\section{Spectral Invariance of $C D_{r e g}$}

We shall show that the spectrum of an element of $C D_{\text {reg }}$ is the same no matter whether it is considered as a bounded operator on $L^{2}(G)$ or as an element of $C D_{\text {reg }}$. To this end we define two representations of $L^{1}(G, R u c(G), T)$ and first show that they are weakly equivalent. The first representation is $R: L^{1}(G, \operatorname{Ruc}(G), T) \rightarrow C D_{\text {reg }} \subset B\left(L^{2}(G)\right.$, which we call the canonical representation. The second one is the $D$-regular representation $\lambda^{D}$ : $L^{1}(G, R u c(G), T) \rightarrow B\left(L^{2}\left(G, L^{2}(G)\right)\right.$, acting on $L^{2}\left(G, L^{2}(G)\right)$.

Proposition 4.1. The representations $\lambda^{D}$ and $R$ of $L^{1}(G, R u c(G), T)$ are weakly equivalent, i.e. $\left\|\lambda^{D}(f)\right\|_{B\left(L^{2}\left(G, L^{2}(G)\right)\right.}=\|R(f)\|_{B\left(L^{2}(G)\right.}$.

Proof. We identify $L^{2}\left(G, L^{2}(G)\right)$ with $L^{2}(G \times G)$. Let $R^{\omega}$ be the extension of $R$ from $L^{2}(G)$ to $L^{2}(G \times G)$ by letting the operators $R(f), f \in \mathcal{L}$, act in the first coordinate only, i.e., for $\xi \in L^{2}(G \times G)$

$$
R^{\omega}(f) \xi(x, z)=\int_{G} f(y)\left(y^{-1} x\right) \xi\left(y^{-1} x, z\right) d y .
$$

Next we define a candidate for an intertwining operator between the $D$ regular representation and the card $(G)$-multiple $R^{\omega}$ of the canonical representation by

$$
S \xi(x, z)=\xi(x z, z), \text { where } \xi \in L^{2}(G \times G) .
$$

Then on the one hand we have

$$
S\left[R^{\omega}(f) \xi\right](x, z)=\int_{G} f(y)\left(y^{-1} x z\right) \xi\left(y^{-1} x z, z\right) d y .
$$

On the other hand

$$
\begin{aligned}
\lambda^{D}(f)(S \xi)(x, z) & =\int_{G}\left(T_{y} f(x y)\right)(z)(S \xi)\left(y^{-1}, z\right) d y \\
& =\int_{G}\left(T_{x^{-1} y} f(y)\right)(z)(S \xi)\left(y^{-1} x, z\right) d y
\end{aligned}
$$




$$
\begin{aligned}
& =\int_{G} f(y)\left(y^{-1} x z\right)(S \xi)\left(y^{-1} x, z\right) d y \\
& =\int_{G} f(y)\left(y^{-1} x z\right) \xi\left(y^{-1} x z, z\right) d y .
\end{aligned}
$$

Consequently,

$$
\lambda^{D}(f)(S \xi)=S R^{\omega}(f) \xi
$$

for all $f \in \mathcal{L}$ and $\xi \in L^{2}(G \times G)$. Since $S$ is unitary on $L^{2}(G \times G), \lambda^{D}$ and $R^{\omega}$ are equivalent, whence $\left\|R^{\omega}(f)\right\|=\left\|\lambda^{D}(f)\right\|$ for all $f \in \mathcal{L}$.

Now for $\xi \in L^{2}(G \times G)$ write $\xi_{z}(x)=\xi(x, z)$ :

$$
\begin{aligned}
\left\|R^{\omega}(f) \xi\right\|^{2} & =\int_{G} \int_{G}\left|\int_{G} f(y)\left(y^{-1} x\right) \xi\left(y^{-1} x, z\right) d y\right|^{2} d x d z \\
& =\int_{G} \int_{G}\left|\int_{G} f(y)\left(y^{-1} x\right) \xi_{z}\left(y^{-1} x\right) d y\right|^{2} d x d z \\
& =\int_{G} \int_{G}\left|R(f) \xi_{z}(x)\right|^{2} d x d z \\
& \leq \int_{G}\left\{\|R(f)\|\left\|\xi_{z}\right\|\right\}^{2} d z \\
& =\|R(f)\|^{2}\|\xi\|^{2} .
\end{aligned}
$$

it follows that $\left\|R^{\omega}(f)\right\| \leq\|R(f)\|$.

For the converse inequality let $\varphi \in L^{2}(G)$ be an element of norm one. Embed $L^{2}(G)$ in $L^{2}\left(G \times G\right.$ by $\xi \mapsto \xi^{\prime}$ where $\xi^{\prime}(x, z)=\xi(x) \varphi(z)$ then

$$
\begin{aligned}
\|R(f) \xi\|^{2} & =\int_{G}|R(f) \xi(x)|^{2} d x \\
& =\int_{G} \int_{G}|R(f) \xi(x)|^{2}|\varphi(z)|^{2} d x d z \\
& =\int_{G} \int_{G}\left|R^{\omega}(f) \xi^{\prime}(x, z)\right|^{2} d x d z \\
& \leq\left\|R^{\omega}(f)\right\|^{2} \int_{G} \int_{G}\left|\xi^{\prime}(x, z)\right|^{2} d x d z \\
& =\left\|R^{\omega}(f)\right\|^{2}\|\xi\|^{2} .
\end{aligned}
$$

Corollary 4.2. Let $G$ be an amenable group, which is rigidly symmetric as a discrete group. Then for $f \in \mathcal{L}$

$$
r_{\mathcal{L}}\left(f^{*} f\right)=\|R(f)\|_{B\left(L^{2}(G)\right)}^{2} .
$$

Proof. We imposed the amenability on $G$ to have that the largest $C^{*}$-norm, denoted $\|\cdot\|_{*}$, on $\mathcal{L}$ is just given by the $D$-regular representation, since the representation $D$ of $R u c$ is a maximal representation [30, Satz 6]. Therefore

$$
\|f\|_{*}=\left\|\lambda^{D}(f)\right\|=\|R(f)\|_{B\left(L^{2}(G)\right)} \quad \forall f \in \mathcal{L} .
$$

Since $\mathcal{L}$ is symmetric Ptaks theorem [38] asserts

$$
r_{\mathcal{L}}\left(f^{*} f\right)=\left\|f^{*} f\right\|_{*}=\|f\|_{*}^{2} .
$$


Theorem 4.3. Let $G$ be an amenable group, which is rigidly symmetric as a discrete group. Then for an operator $A \in C D_{\text {reg }}$

$$
s p_{C D}(A)=s p_{B\left(L^{2}(G)\right)}(A) .
$$

Proof. Since $A=R(f)$ for some $f \in \mathcal{L}$, this follows from the above corollary by an application of Hulanicki's Lemma [15].

\section{An Example of Non Symmetry}

A special role in the theory of symmetry of group algebras is played by the group of affine mappings of the real line, the " $a x+b$ "-group. This group has a symmetric $L^{1}$-algebra [33], but its discretised version is not symmetric [23]. We do not know about the rigid symmetry of the continuous group. We shall consider the connected component of the identity.

$$
E=\left\{\left(\begin{array}{ll}
a & b \\
0 & 1
\end{array}\right), \quad a>0, \quad b \in \mathbb{R}\right\}
$$

The multiplication is $(a, b) \cdot\left(a^{\prime}, b^{\prime}\right)=\left(a a^{\prime}, a b^{\prime}+b\right)$ and the action on $\mathbb{R}$ : $(a, b): x \mapsto a x+b$. The left Haar measure is $\frac{d a}{a^{2}} d b$.

Theorem 5.1. For this group $L^{1}(E, R u c(E), T)$ is not symmetric, where $T$ is left translation on the right uniformly continuous functions $R u c(E)$.

Proof. We identify the normal subgroup $N$ of translations with $\mathbb{R}$, and by means of the exponential map identify $\mathbb{R}$ with the subgroup of dilations.

Explicitly Let $\omega:(s, x) \mapsto e^{s} x$ from $\mathbb{R} \times \mathbb{R} \rightarrow \mathbb{R}$. The action of $\mathbb{R}$ on $C_{0}(\mathbb{R})$ is given by $u \mapsto u^{s}:=u(\omega(s, \cdot))$. The additive group $\mathbb{R}$ acts on $L^{1}\left(\mathbb{R}, C_{0}(\mathbb{R})\right)$ by

$\tilde{T}(s) f(x)(\cdot)=e^{-s} f(\omega(-s, x))(\omega(s, \cdot)) \in C_{0}(\mathbb{R}), \quad f \in L^{1}\left(\mathbb{R}, C_{0}(\mathbb{R})\right), s, x \in \mathbb{R}$.

Here $e^{s}$ is the modulus of the action $\omega(s, \cdot)$ on $\mathbb{R}$, with respect to translation invariant Lebesgue measure $d x$. From [35, Theorem 5] we know that the twisted algebra $L^{1}\left(\mathbb{R}, L^{1}\left(\mathbb{R}, C_{0}(\mathbb{R})\right), \tilde{T}\right)$ is not symmetric. So the theorem will be proved if we show that this Banach-* algebra is isomorphic to a closed *-subalgebra of $L^{1}(E, R u c(E), T)$.

We let $E$ act on $C_{0}(\mathbb{R})$ by $T^{\prime}((a, b)): u \mapsto u \circ \omega(\log (a), \cdot)$. Then $L^{1}\left(E, C_{0}(\mathbb{R}), T^{\prime}\right)$ and $L^{1}\left(\mathbb{R}, L^{1}\left(\mathbb{R}, C_{0}(\mathbb{R})\right), \tilde{T}\right)$ are isometrically isomorphic Banach *-algebras: For $f \in L^{1}\left(\mathbb{R}, L^{1}\left(\mathbb{R}, C_{0}(\mathbb{R})\right), \tilde{T}\right)$ let $S f \in L^{1}\left(E, C_{0}(\mathbb{R}), T^{\prime}\right)$ be defined by $S f(a, b)=f(\log (a))\left(-\frac{b}{a}\right) \in C_{0}(\mathbb{R}), \quad(a, b) \in E$. Indeed

$$
\|S f\|=\int_{\mathbb{R}} \int_{\mathbb{R}_{>0}}\|S f(a, b)\|_{\infty} \frac{d a}{a^{2}} d b=\int_{\mathbb{R}} \int_{\mathbb{R}}\|f(s)(b)\|_{\infty} d s d b=\|f\| .
$$


With the notation $a^{\prime}=e^{t}$ and $a=e^{s}$ :

$$
\begin{aligned}
& S(f \stackrel{\tilde{T}}{*} h)(a, b)=(f \stackrel{\tilde{T}}{*} h)(s)\left(-\frac{b}{a}\right)=\int_{\mathbb{R}}(\tilde{T}(t) f(s+t)) * h(-t)\left(-\frac{b}{a}\right) d t \\
& \left.=\int_{\mathbb{R}} \int_{\mathbb{R}}(\tilde{T}(t) f(s+t))\left(\frac{-b}{a}+v\right) \cdot h(-t)\right)(-v) d v d t \\
& \left.=\int_{\mathbb{R}} \int_{\mathbb{R}} e^{-t} f(s+t)\left(\omega\left(-t,-\frac{b}{a}+v\right)\right) \circ \omega(t, \cdot)\right) \cdot \\
& h(-t))(-v) d v d t \\
& \left.=\int_{\mathbb{R}} \int_{\mathbb{R}} e^{-t} f(s+t)\left(\omega\left(-t,-\frac{b}{a}-v\right)\right) \circ \omega(t, \cdot)\right) \cdot \\
& h(-t))(v) d v d t \\
& \left.\left.=\int_{\mathbb{R}} \int_{\mathbb{R}} e^{-t} f(s+t)\left(-e^{-t}\left(\frac{b}{a}+v\right)\right)\right) \circ \omega(t, \cdot)\right) \text {. } \\
& h(-t))(v) d v d t \\
& =\int_{\mathbb{R}} \int_{\mathbb{R}} e^{-t} S f\left(e^{s+t}, e^{s}\left(\frac{b}{a}+v\right)\right) \circ \omega(t, \cdot) \text {. } \\
& \operatorname{Sh}\left(e^{-t},-e^{-t} v\right) d v d t \\
& =\int_{\mathbb{R}} \int_{\mathbb{R}_{>0}} S f\left(a a^{\prime}, a\left(\frac{b}{a}+v\right)\right) \circ \omega\left(\log \left(a^{\prime}\right), \cdot\right) \\
& \cdot S h\left(\frac{1}{a^{\prime}},-\frac{v}{a^{\prime}}\right) \frac{d a^{\prime}}{a^{\prime 2}} d v \\
& =\int_{\mathbb{R}} \int_{\mathbb{R}_{>0}} S f\left((a, b)\left(a^{\prime}, v\right)\right) \circ \omega\left(\log \left(a^{\prime}\right), \cdot\right) \operatorname{Sh}\left(\left(a^{\prime}, v\right)^{-1}\right) \frac{d a^{\prime}}{a^{\prime 2}} d v \\
& =\int_{\mathbb{R}} \int_{\mathbb{R}_{>0}} T^{\prime}\left(\left(a^{\prime}, v\right) S f\left((a, b)\left(a^{\prime}, v\right)\right) \operatorname{Sh}\left(\left(a^{\prime}, v\right)^{-1}\right) \frac{d a^{\prime}}{a^{\prime 2}} d v\right. \\
& =\left(S f \stackrel{T^{\prime}}{*} S h\right)(a, b),
\end{aligned}
$$

For the $*$ operation on the respective algebras we have

$$
(S f)^{*}((a, b))=\Delta_{E}\left((a, b)^{-1}\right) T^{\prime}\left((a, b)^{-1}\right) \overline{S f\left((a, b)^{-1}\right)},
$$

and

$$
f^{*}(s)(b)=\tilde{T}(-s) \overline{f(-s)(-b)}
$$

So that

$$
\begin{aligned}
& f^{*}(s)(b)=\tilde{T}(-s)(\overline{f(-s)(-b)}
\end{aligned}
$$

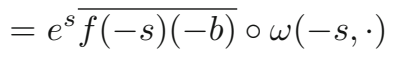

$$
\begin{aligned}
& =\overline{a S f\left(\frac{1}{a},-\frac{b}{a}\right)} \circ \omega\left(\log \left(\frac{1}{a}, \cdot\right)\right. \\
& =\Delta_{E}\left(\left(a^{-1},-\frac{b}{a}\right)\right) T^{\prime}\left(\left(a^{-1},-\frac{b}{a}\right)\right) \overline{S f\left(\left(a^{-1},-\frac{b}{a}\right)\right)}
\end{aligned}
$$




$$
\begin{aligned}
& =\Delta_{E}\left((a, b)^{-1}\right) T^{\prime}\left((a, b)^{-1}\right) \overline{S f\left((a, b)^{-1}\right)} \\
& =(S f)^{*}((a, b)) .
\end{aligned}
$$

Now we can embed $C_{0}(\mathbb{R}) \rightarrow R u c(E)$ by extending a function, already defined on the dilations to a function on $E$ which is independent of the translation coordinate of a group element. Restricting the left translation to this subspace is just the action we denoted $T^{\prime}$. So we obtain an embedding of $L^{1}\left(E, C_{0}(\mathbb{R}), T^{\prime}\right)$ into $L^{1}(E, R u c(E), T)$.

Corollary 5.2. $C D_{\text {reg }}(E)$ is not inverse-closed in $B\left(L^{2}(E)\right)$.

Proof. By the above theorem there is a selfadjoint operator in $C D_{\text {reg }}(E)$, with a non-real spectrum. So some operator in $C D_{\text {reg }}(E)+\mathbb{C}$ id is invertible in $B\left(L^{2}(E)\right)$ but not in $C D_{\text {reg }}(E)+\mathbb{C i d}$.

\section{Acknowledgements}

Open access funding provided by [University of Vienna].

We are grateful to the University of Vienna for this.

Open Access. This article is distributed under the terms of the Creative Commons Attribution 4.0 International License (http://creativecommons.org/licenses/ by/4.0/), which permits unrestricted use, distribution, and reproduction in any medium, provided you give appropriate credit to the original author(s) and the source, provide a link to the Creative Commons license, and indicate if changes were made.

\section{References}

[1] Balan, R.: The noncommutative Wiener lemma, linear independence. and spectral properties of the algebra of time-frequency shift operators. Trans. Am. Math. Soc. 360(7), 3921-3941 (2008)

[2] Baskakov, A.G.: Wiener's theorem and asymptotic estimates for elements of inverse matrices. Funktsional. Anal. i Prilozhen. 24(3), 64-65 (1990)

[3] Baskakov, A.G.: Abstract harmonic analysis and asymptotic estimates for elements of inverse matrices. Mat. Zametki 52(2), 17-26, 155 (1992)

[4] Baskakov, A.G.: Asymptotic estimates for elements of matrices of inverse operators, and harmonic analysis. Sibirsk. Mat. Zh., 38(1), 14-28, i (1997)

[5] Baskakov, A.G.: Estimates for the elements of inverse matrices, and the spectral analysis of linear operators. Izv. Ross. Akad. Nauk Ser. Mat. 61(6), 3-26 (1997)

[6] Beltiţă, I., Beltiţă, D.: Erratum to: Inverse-closed algebras of integral operators on locally compact groups. Ann. Henri Poincaré 16(5), 1307-1309 (2015)

[7] Beltiţă, I., Beltiţă, D.: Inverse-closed algebras of integral operators on locally compact groups. Ann. Henri Poincaré 16(5), 1283-1306 (2015)

[8] Bochner, S., Phillips, R.S.: Absolutely convergent Fourier expansions for noncommutative normed rings. Ann. Math 43(2), 409-418 (1942)

[9] Leeuw, K.De : An harmonic analysis for operators. I. Formal properties. Ill. J. Math. 19(4), 593-606 (1975)

[10] Diestel, J., Uhl Jr., J.J.: Vector Measures. American Mathematical Society, Providence, RI (1977) 
[11] Dixmier, J.: $C^{*}$-algebras. Translated from the French by Francis Jellett. NorthHolland Publishing Co., Amsterdam, New York, Oxford (1977)

[12] Farrell, B., Strohmer, T.: Inverse-closedness of a Banach algebra of integral operators on the Heisenberg group. J. Oper. Theory 64(1), 189-205 (2010)

[13] Fendler, G., Gröchenig, K., Leinert, M.: Convolution-dominated operators on discrete groups. Integral Equations Oper. Theory 61(4), 493-509 (2008)

[14] Fendler, G., Gröchenig, K., Leinert, M.: Convolution-dominated integral operators. In: Noncommutative harmonic analysis with applications to probability II, vol. 89 of Banach Center Publ., pp. 121-127. Polish Acad. Sci. Inst. Math., Warsaw (2010)

[15] Fendler, G., Groechenig, K.M Leinert: Symmetry of weighted $L^{1}$-algebras and the GRS-condition. Bull. Lond. Math. Soc. 38(4), 625-635 (2006)

[16] Gohberg, I., Kaashoek, M.A., Woerdeman, H.J.: The band method for positive and strictly contractive extension problems: an alternative version and new applications. Integral Equations Oper. Theory 12(3), 343-382 (1989)

[17] Gröchenig, K.: Time-frequency analysis of Sjöstrand's class. Rev. Mat. Iberoam. 22(2), 703-724 (2006)

[18] Gröchenig, K., Klotz, A.: Noncommutative approximation: inverse-closed subalgebras and off-diagonal decay of matrices. Constr. Approx. 32(3), 429466 (2010)

[19] Gröchenig, K., Klotz, A.: Norm-controlled inversion in smooth Banach algebras. I. J. Lond. Math. Soc. 88(1), 49-64 (2013)

[20] Gröchenig, K., Klotz, A.: Norm-controlled inversion in smooth Banach algebras, II. Math. Nachr. 287(8-9), 917-937 (2014)

[21] Gröchenig, K., Leinert, M.: Wiener's lemma for twisted convolution and Gabor frames. J. Am. Math. Soc. 17(1), 118 (2004)

[22] Hulanicki, A.: On the spectrum of convolution operators on groups with polynomial growth. Invent. Math. 17, 135-142 (1972)

[23] Jenkins, J.W.: An amenable group with a non-symmetric group algebra. Bull. Am. Math. Soc. 75, 357-360 (1969)

[24] Klotz, A.: Spectral invariance of Besov-Bessel subalgebras. J. Approx. Theory 164(2), 268-296 (2012)

[25] Klotz, A.: Inverse closed ultradifferential subalgebras. J. Math. Anal. Appl. 409(2), 615-629 (2014)

[26] Kurbatov, V.G.: Functional-differential operators and equations, vol. 473 of Mathematics and its Applications. Kluwer Academic Publishers, Dordrecht (1999)

[27] Kurbatov, V.G.: Some algebras of operators majorized by a convolution. Funct. Differ. Equ. 8(3-4), 323-333 (2001) [International Conference on Differential and Functional Differential Equations (Moscow 1999)]

[28] Leptin, H.: Verallgemeinerte $L^{1}$-Algebren und projektive Darstellungen lokal kompakter Gruppen I. Invent. Math. 3, 257-281 (1967)

[29] Leptin, H.: Verallgemeinerte $L^{1}$-Algebren und projektive Darstellungen lokal kompakter Gruppen II. Invent. Math 4, 68-86 (1967)

[30] Leptin, H.: Darstellungen verallgemeinerter $L^{1}$-Algebren. Invent. Math 5, 192215 (1968) 
[31] Leptin, H.: Ideal theory in group algebras of locally compact groups. Invent. Math. 31, 259-278 (1976)

[32] Leptin, H.: Symmetrie in Banachschen Algebren. Arch. Math. (Basel) 27(4), 394-400 (1976)

[33] Leptin, H.: Lokal kompakte Gruppen mit symmetrischen Algebren. In: Symposia Mathematica, vol. XXII (Convegno sull'Analisi Armonica e Spazi di Funzioni su Gruppi Localmente Compatti, INDAM, Rome 1976), pp. 267-281. Academic Press, London (1977)

[34] Leptin, H.: On one-sided harmonic analysis in noncommutative locally compact groups. J. Reine Angew. Math. 306, 122-153 (1979)

[35] Leptin, H., Poguntke, D.: Symmetry and non-symmetry for locally compact groups. J. Funct. Anal. 33, 119-134 (1979)

[36] Măntoiu, M.: Symmetry and inverse closedness for Banach *-algebras associated to discrete groups. Banach J. Math. Anal. 9(2), 289-310 (2015)

[37] Poguntke, D.: Rigidly symmetric $L^{1}$-group algebras. Sem. Sophus Lie 2, 189197 (1992)

[38] Pták, V.: On the spectral radius in Banach algebras with involution. Bull. Lond. Math. Soc. 2, 327-334 (1970)

[39] Reiter, H.: Classical harmonic analysis and locally compact groups. Oxford University Press, (1967)

[40] Schep, A.R.: Kernel operators. Rijksuniversiteit te Leiden, Leiden (1977)

[41] Shin, C.E., Sun, Q.: Wiener's lemma: localization and various approaches. Appl. Math. J. Chinese Univ. Ser. B 28(4), 465-484 (2013)

[42] Sjöstrand, J.: Wiener type algebras of pseudodifferential operators. In: Séminaire sur les Équations aux Dérivées Partielles, 1994-1995, Exp. No. IV. École Polytech., Palaiseau (1995)

[43] Sun, Q.: Wiener's lemma for infinite matrices. Trans. Am. Math. Soc. 359(7), 3099-3123 (2007) (electronic)

[44] Wiener, N.: Tauberian theorems. Ann. Math. 33(1), 1-100 (1932)

Gero Fendler $(\bowtie)$

Fakultät für Mathematik

Universität Wien

Oskar-Morgenstern-Platz 1

1090 Wien

Austria

e-mail: gero.fendler@univie.ac.at

Michael Leinert

Institut für Angewandte Mathematik

Universität Heidelberg

Im Neuenheimer Feld 294

69120 Heidelberg

Germany

e-mail: leinert@math.uni-heidelberg.de

Received: March 10, 2016.

Revised: September 19, 2016. 